\begin{tabular}{|c|c|}
\hline $\begin{array}{l}\text { Una historia sepultada: el Centro de Estudios Socioeconómicos de la Universidad de } \\
\text { Chile, 1965-1973 (a } 50 \text { años de su fundación) }\end{array}$ & Titulo \\
\hline Cárdenas Castro, Juan Cristóbal - Autor/a; & Autor(es) \\
\hline $\begin{array}{l}\text { De Raíz Diversa. Revista Especializada en Estudios Latinoamericanos (Vol. } 2 \text { no. } 3 \\
\text { ene-jun 2015) }\end{array}$ & En: \\
\hline México D.F. & Lugar \\
\hline $\begin{array}{l}\text { Programa de Posgrado en Estudios Latinoamericanos, Universidad Nacional } \\
\text { Autónoma de México }\end{array}$ & Editorial/Editor \\
\hline \multirow[t]{2}{*}{2015} & Fecha \\
\hline & Colección \\
\hline $\begin{array}{l}\text { Teoría de la dependencia; Transición política; Socialismo; CESO, Centro de Estudios } \\
\text { Socioeconómicos; Universidad de Chile; Intelectuales; Exilio; Chile; América Latina; }\end{array}$ & Temas \\
\hline Artículo & Tipo de documento \\
\hline $\begin{array}{l}\text { "http://biblioteca.clacso.edu.ar/Mexico/ppel-unam/20160621043024/Cardenas_Juan_Cristobal_Una_historia_sepultada_el_Centro_de_E } \\
\text { studios_Socioeconomicos_de_la_Universidad_de_Chile_1965-1973_a_50_aNos_de_su_fundacion.pdf" }\end{array}$ & URL \\
\hline $\begin{array}{l}\text { Reconocimiento-No Comercial-Sin Derivadas CC BY-NC-ND } \\
\text { http://creativecommons.org/licenses/by-nc-nd/2.0/deed.es }\end{array}$ & Licencia \\
\hline
\end{tabular}

\begin{tabular}{|c|}
\hline $\begin{array}{c}\text { Segui buscando en la Red de Bibliotecas Virtuales de CLACSO } \\
\text { http://biblioteca.clacso.edu.ar }\end{array}$ \\
Consejo Latinoamericano de Ciencias Sociales (CLACSO) \\
Conselho Latino-americano de Ciências Sociais (CLACSO) \\
Latin American Council of Social Sciences (CLACSO) \\
www.clacso.edu.ar \\
\end{tabular}
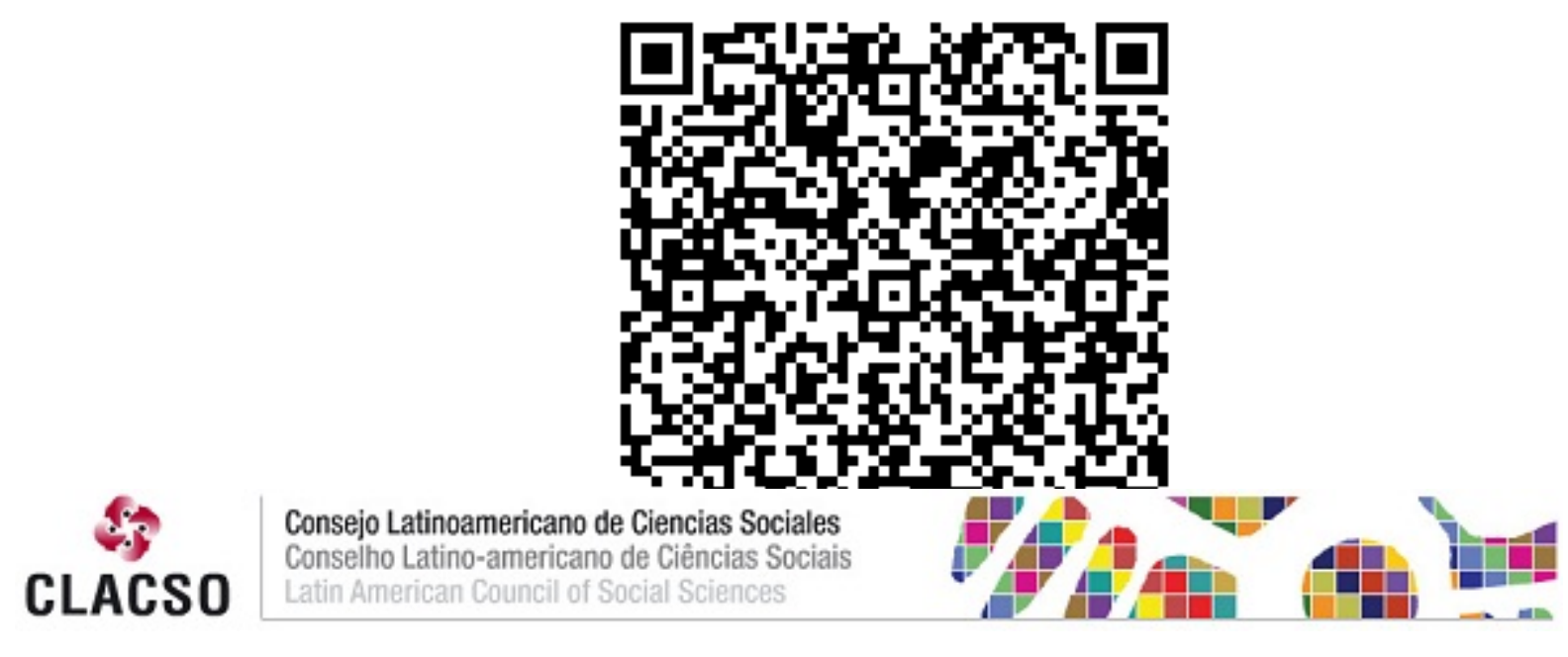


\title{
Una historia sepultada: el Centro de Estudios Socioeconómicos de la Universidad de Chile, 1965-1973 (a 50 años de su fundación)*
}

\author{
Juan Cristobal Cárdenas Castro**
}

RESUMEN: Entre 1965 y 1973, tuvo lugar una interesante experiencia de trabajo intelectual y construcción de pensamiento crítico en Latinoamérica, en el Centro de Estudios Socioeconómicos (CESO), de la Universidad de Chile. $\mathrm{Su}$ actividad se vio potenciada a partir de 1966 con la incorporación de varios académicos que se habían exiliado tras la persecución política desatada por los golpes de Estado y la intervención militar de las universidades en diversos países de la región. Con la llegada de Theotonio Dos Santos, Vania Bambirra, Ruy Mauro Marini, Emir Sader, Edimilson Bizelli, Tomás Amadeo Vasconi y -por motivos diferentes- André Gunder Frank, que se sumaron al grupo de jóvenes egresados de la Facultad de Ciencias Económicas reclutados por el sociólogo Eduardo Hamuy -entre ellos, Orlando Caputo, Roberto Pizarro, Sergio Ramos, Pío García y Cristóbal Kay-, fueron creados varios equipos de investigación, entre los que destaca el dedicado al estudio de las relaciones de dependencia en América Latina. El esfuerzo teórico desplegado por los investigadores del CESO, que dio algunos de sus más notables desarrollos durante el gobierno de la Unidad Popular, fue interrumpido -y ese Centro definitivamente clausurado- tras el golpe militar que derrocó a Salvador Allende.

Palabras Clave: Exilio intelectual, teoría de la dependencia, transición al socialismo.

Abstrac. Between 1965 and 1973, there was an interesting experience of intellectual work and construction of critical thinking in Latin America, in the Center for Socioeconomic Studies (known by its Spanish acronym, CEso) of the University of Chile. Its activity was enhanced from 1966 with the addition of several scholars who were exiled after the political persecution by coups and military intervention of universities in various countries of the region. With the arrival of Theotonio Dos Santos, Vania Bambirra, Ruy Mauro Marini, Emir Sader, Edimilson Bizelli, Tomás Amadeo Vasconi and -for other reasons-André Gunder Frank, who joined the group of young graduates from the Faculty of Economics recruited by sociologist Eduardo Hamuy -including Orlando

\footnotetext{
Versión corregida y ampliada del trabajo que presentamos al xxix Congreso de la Asociación Latinoamericana de Sociología, realizado en Santiago de Chile del 29 de septiembre al 4 de octubre de 2013 .

** Candidato a Doctor en el Programa de Posgrado en Estudios Latinoamericanos de la UNAM.
} 
Caputo, Roberto Pizarro, Sergio Ramos, Pío Garcia and Cristobal Kay- were created several research teams, most notably the one dedicated to the study of dependency relations in Latin America. The theoretical effort made by researchers of CESO, who gave some of his most notable developments during the Unidad Popular government, was interrupted -and that center definitely closed- after the military coup that overthrew Salvador Allende.

KEYWORDS: Intellectual exile, dependency theory, transition to socialism.

Recibido: 26 de septiembre de 2014. ACEPTAdo: 29 de octubre de 2014 .

El CESo fue, en su momento, uno de los principales centros intelectuales de América

Latina. La mayoría de la intelectualidad latinoamericana, europea y estadounidense, principalmente de izquierda, pasó por ahí, participando mediante charlas, conferencias, mesas redondas y seminarios. Sin embargo, el secreto de la intensa vida intelectual que lo caracterizó y que se constituyó en la fuente real de su prestigio fue la permanente práctica

interna de diálogo y discusión, institucionalizada en los seminarios de área-las áreas temáticas eran las células de la institución-, en el seminario general, y continuada en las relaciones personales, que tenían por base el compañerismo y el respeto recíproco.

Ruy Mauro Marini, c1990.

\section{PREÁMBULO}

A

penas habían transcurrido algunas pocas semanas desde la apertura del Centro y en el decanato de la entonces Facultad de Ciencias Económicas de la Universidad de Chile, ataviados de esa vieja manía de ponerle siglas a todo, ya lo habían bautizado como 'CESEC'. "Pero nosotros no nos llamamos así”, sentenció don Eduardo. Y se quedó pensando, lápiz y papel en mano. Evidentemente le preocupaba que esa denominación tan poco imaginativa terminara generalizándose. A Pío, el joven egresado de la Escuela de Economía que estaba sentado frente a él, le habrá parecido excesiva la importancia que su interlocutor le concedía al asunto. Si bien el anhelo de fundar una nueva institución científica que cumpliera cabalmente su función investigadora de la realidad social se había hecho posible, don Eduardo no parecía dispuesto a que se le colocaran unas siglas sin sentido que consideraba excesivamente convencionales. 'CESO', escribió después. Y manifiestamente complacido sentenció: "Nosotros somos la parte pensante de esta Facultad, somos el 'seso' de la Facultad. Así que por favor comunícale al decanato que en todas partes que se refieran a nosotros digan Ceso". Así fue como en los primeros meses de 1965 bautizó al 
Centro de Estudios Socioeconómicos su director-fundador, el sociólogo Eduardo Hamuy, según recuerda Pío García, quien había asumido como investigador y secretario ejecutivo (García, 2012/2013).

El hecho puede considerarse sintomático de las proyecciones con las que surgió el Centro, y que animaron su desarrollo. Y es que sin dudarlo, su creación se convertiría en uno de los resultados más importantes de la reforma académica realizada en la Facultad de Ciencias Económicas hacia fines de 1964, en tiempos de la contienda presidencial de la que resultó vencedor -bajo la promesa de impulsar una 'Revolución en libertad'Eduardo Frei Montalva, entusiasta partidario de la controvertida 'Alianza para el Progreso' impulsada por J. F. Kennedy.

En las líneas siguientes nos proponemos examinar algunos aspectos destacados de la 'biografía' del CESO. Su historia quedó prácticamente sepultada tras el sangriento golpe cívico-militar del 11 de septiembre de 1973. Desde entonces, poco se ha publicado en relación con lo que ese Centro representó y cimentó durante sus casi ocho años de existencia. Valgan las reflexiones siguientes como una contribución al desentierro de un fragmento de la historia intelectual de nuestro país o, mejor aún, de un girón del pensamiento crítico latinoamericano, ya que es un episodio cruzado -en gran medida- por el exilio intelectual tras los golpes de Estado que asolaron la región entre 1964 y 1973. Para ello, dividimos nuestra exposición en dos partes, que identifican dos grandes momentos en la historia de aquel centro. En la primera, ponemos atención a los objetivos que inspiraron la creación del Ceso, así como a los aciertos y dificultades de esos primeros años. En la segunda, damos cuenta de la trascendencia alcanzada por ese centro, especialmente durante el gobierno de la Unidad Popular (1970-1973). A modo de conclusión, finalizamos este ensayo con unas notas relativas a su clausura y a la persecución política y académica de la que fueron objeto sus investigadores.

PRIMEROS AÑOS Y AVATARES (1965 - 1968)

Aunque el CESO inició formalmente sus actividades en enero de 1965 en el $4^{\circ}$ piso de la Escuela de Economía ubicada en República 517, prontamente se trasladó a la casa de la calle Gay 2360 en Santiago Centro y al año siguiente a su residencia definitiva en la antigua casona de Av. España 620, ubicada a pocas cuadras del edificio de la Escuela de Economía y sus 
pensionados, que de hecho conforman el origen del actual barrio universitario de República.

Desde su puesta en marcha "el CEso se constituyó en centro de referencia institucional de las corrientes de pensamiento crítico dentro de la Facultad, en mucho animadas por quien fuera su fundador y primer director, Eduardo Hamuy" (García, 1980: 82). Y es que el optimismo despertado en América Latina tras el triunfo de la revolución cubana en 1959, motivó que en Chile el movimiento estudiantil reapareciera en la primera línea de la disputa política, llegando a ser, entre otros, un factor importante de la victoria en el país de Frei, en 1964, y desde una orientación muy distinta, de la creación en Concepción del Movimiento de Izquierda Revolucionaria (MIR), en 1965. En el caso de la Escuela de Economía, desde comienzos de los sesenta, los estudiantes se habían movilizado para exigir una reforma de los contenidos académicos de los planes de estudio, consiguiendo la apertura de diversos cursos para el estudio de temáticas hasta entonces apenas consideradas, como las concepciones desarrollistas de la CEPAL, o simplemente ignoradas, como el marxismo, así como de otras disciplinas -entre ellas: sociología, historia social, psicología social, ciencia política- que permitieran una formación más integral de los economistas. Expresión del signo de la reforma académica aprobada en aquella Facultad en octubre de 1964, es la creación del CESo, ya que:

En contraposición a los contenidos analíticos y disociadores de la realidad social que imperaban en la docencia y la investigación de la Facultad, el objetivo definido del Centro era el estudio científico integrado del proceso histórico de desarrollo y cambio social (García, 1980: 82).

En gran medida ese objetivo fue expuesto con claridad por Hamuy en un seminario realizado en el Instituto de Economía de la Universidad de Chile en junio de 1965. En una breve exposición titulada "Historiar el presente", su autor explica los fundamentos del CESO, además de las razones consideradas para adoptar la línea de investigación en cuestión:

Nuestro punto de partida, y realmente lo que hubo detrás del proyecto de creación de este Centro, fue la necesidad que tiene el país de que existan instituciones que se preocupen de los grandes problemas nacionales con un criterio macrosocial (Hamuy, 1965:105).

De acuerdo con Hamuy, cada proceso de cambio social trae aparejado un reajuste de carácter institucional y una redistribución del poder o, 
de otra manera, un cambio en la estructura del poder de una sociedad. Y Chile estaba comenzando a vivir bajo un signo que le confería al fenómeno social un especial interés. Se trataba de un momento único que tenía todas las características de un gran experimento social, cuyo estudio no debía ser desperdiciado desde ninguno de sus aspectos -económicos, sociológicos, psicológicos-sociales, etc.-, pues en todos ellos se abría un campo insospechado de investigación. Anotaba que:

...no sabemos que haya existido en alguno de los actuales países industriales una institución que en el momento crítico se haya preocupado conscientemente de estudiar este proceso de una manera sistemática y científica. Este es el papel que nos hemos asignado: estudiar los procesos fundamentales que están ocurriendo actualmente en Chile. Intentar ser los historiadores del presente. La tarea que nos hemos impuesto tratamos de cumplirla utilizando un enfoque global o como se le llama, interdisciplinario (Hamuy, 1965: 108).

Y agregaba que:

el análisis científico de esta etapa que ha iniciado Chile, es en el fondo, el estudio del comportamiento de los factores económicos y políticos que influyen en el proceso de cambios sociales... La finalidad básica del Centro de Estudios Socio Económicos es la investigación y conocimiento del proceso de cambios sociales. (Hamuy, 1965: 109)

Además, meses más tarde Hamuy señalaría que uno de los pendientes de las universidades chilenas era "la tarea de colaborar junto al pueblo... con el propósito de encontrar las mejores soluciones a los grandes problemas sociales" (Hamuy, 1966: s/n). Y manifestaba que:

En el pueblo se está cuando sus problemas constituyen la preocupación central, cuando la investigación se convierte en un acto de humildad, en un estar para aprender del pueblo, para sistematizar su rica experiencia, para racionalizar sus visiones y para organizar conceptualmente sus intuiciones. La investigación científico-social es, en consecuencia, un acto de humildad en cuanto se parte de la base que es del pueblo de donde hay que aprender para devolver en enseñanza lo que se recibe de él, después de disciplinar la experiencia con rigor científico. Este[...] diálogo permanente con el pueblo, es la condición necesaria del progreso de las ciencias sociales (Hamuy, 1966: $\mathrm{s} / \mathrm{n}$ ).

Una nítida preocupación expresaba igualmente al constatar que en la Universidad de Chile se acentuaba la elitización y que mientras el país se 
había ido democratizando, ella experimentaba un proceso inverso, "había dejado de ser la institución que antaño reforzaba la democracia reduciendo las diferencias sociales" (Hamuy, 1966: s/n) y, por el contrario, contribuía a reforzar el sistema oligárquico de la sociedad chilena. Sin duda, esta apreciación de Hamuy fue una advertencia de los problemas que atravesaban las universidades chilenas y que se hicieron del todo evidentes tras el estallido de la llamada 'reforma universitaria' en junio de 1967. Asimismo, resulta de interés su mirada crítica del rol de la investigación científica en las universidades y la constatación de la cada vez mayor dependencia académica creada por las donaciones y ayudas extranjeras, que consideraba como fuentes de 'alienación científica'.

La creación del CESO es fruto de esas inquietudes expresadas por Hamuy y que fueron las mismas preocupaciones de una generación de científicos sociales que emergió con fuerza en América Latina en la década de los sesenta. Y así como desde un inicio este Centro llegó a ser un referente de las corrientes de pensamiento crítico en la Facultad de Ciencias Económicas, pronto consiguió rebasar con creces esa demarcación, ayudado en gran medida por la solidaria recepción que la Universidad de Chile hizo de cientos de científicos sociales latinoamericanos exonerados de sus universidades tras los golpes militares que, en apego a la 'Doctrina de Seguridad Nacional', fueron promovidos por el gobierno de los Estados Unidos (Comblin, 1978).

En este contexto, uno de los grandes aciertos en la trayectoria del CESo es la temprana incorporación, en el segundo trimestre de 1966, de un joven sociólogo brasileño, recomendado a Hamuy por su colega Florestán Fernándes. Theotonio Dos Santos llegaba exiliado al país tras ser expulsado de la Universidad de Brasilia y pasar dos años clandestino en São Paulo, perseguido por su militancia política, y luego ser condenado por un tribunal militar a 15 años de presidio.

Aunque es poco probable que hayan existido mutuas y tempranas influencias entre la reflexión que comenzaba a desarrollar Dos Santos y las ideas de Hamuy, podemos sostener que, tal como fue concebido el proyecto del CESO, hubo profundas coincidencias en las apreciaciones que sobre varios temas tenían ambos sociólogos, seguramente motivadas por similares diagnósticos sobre las posibilidades de cambio social abiertas en América Latina, especialmente tras el triunfo de la Revolución Cubana. Lo cierto es que la llegada de Dos Santos insufló nueva creatividad 
al CESo, que en sus primeros años se había abocado principalmente "a la realización de encuestas de opinión y actitudes [en las que Hamuy venía trabajando desde 1957] en la mejor tradición de la sociología empírica norteamericana" (García, 1980), y de algunas investigaciones de carácter histórico (Góngora, 1966; Hernández, 1966; Ladrón de Guevara, 1967; Izquierdo, 1968; Carmagnani, 1971) y y sociológico (Hamuy, 1966 y 1967; Descouvières, 1968).

Tras organizar a fines de 1966 un exitoso seminario sobre clases sociales, Dos Santos creó en el CESo, en junio de 1967, un equipo de investigación sobre las relaciones de dependencia en América Latina, la más importante línea de estudio desarrollada en ese Centro, al que se integraron tres jóvenes egresados de la Escuela de Economía: Orlando Caputo, Roberto Pizarro y Sergio Ramos. Al poco tiempo, al equipo se sumó la socióloga brasileña Vania Bambirra, mientras se contaba también con la colaboración de algunos becarios de nacionalidad peruana. Aún se conserva el esquema y plan de trabajo que orientó los primeros pasos de este equipo de investigación (CESO, 1967). Junto con una recopilación bibliográfica y el estudio de "las principales contribuciones a la teoría de la dependencia", que cristalizaron en dos de los primeros trabajos colectivos desarrollados por este equipo (Santos, T.; Bambirra, V.; Caputo, O.; Martínez, J.; Pizarro, R. \& Ramos, S., 1968 y 1969), se analizó el impacto de la inversión exterior en América Latina, los tipos de dependencia por grupos de países y la evolución histórica de la estructura del capital extranjero en la región. Por otra parte, el trabajo del equipo permitió que los jóvenes investigadores elaboraran sus memorias de grado, destacando dos excelentes trabajos publicados en la serie Cuadernos del CESO; nos referimos a la investigación de Caputo y Pizarro titulada Imperialismo, dependencia y relaciones económicas internacionales (1971), y a la de Ramos, que llevó por nombre Chile: ¿una economía de transición? (1972).

Destaca, además, la extensa producción teórica que pronto perfiló a Dos Santos como un sociólogo de envergadura. De esta primera época

\footnotetext{
1 Esta última investigación, publicada en Italia algo más tarde que las anteriores, se encontraba lista para su publicación en castellano al momento en que sobrevino el golpe militar de 1973. Lo mismo aconteció con varias investigaciones realizadas en el CESO en los últimos años, algunas de las cuales fueron publicadas por sus autores tras la clausura del mismo, entre ellas: Valenzuela, 1974; Sánchez, 1976; Kay, 1980; Carmagnani, 1998. No sucedió lo mismo con el que iba a ser el Cuaderno $n^{\circ} 17$ del CESO, la traducción de la investigación de Alexander Schejtman (1970).
} 
son sus ensayos sobre El nuevo carácter de la dependencia (1967 y 1968b), Crisis de la Teoría del Desarrollo y las relaciones de dependencia en América Latina (1968c), Socialismo o fascismo: dilema latinoamericano (1969b) y Dependencia y cambio social (1970).

Por otra parte, esa producción se potenció con la llegada al CESo del filósofo Tomás Amadeo Vasconi, a fines de 1967, quien desde temprano -junto con su compañera Inés Reca- se dedicó al estudio sistemático de la reforma en la Universidad de Chile, además de legarnos algunos valiosos ensayos sobre Educación y cambio social (1967), Dependencia y superestructura (1970) y Modernización y crisis en la universidad latinoamericana (1971).

Finalmente, resulta conveniente hacer mención de dos hechos. El primero es que a fines de octubre de 1966, tras haberse titulado como economista con una tesis de grado basada en una investigación realizada en el CESo sobre propensión al cambio entre los funcionarios públicos, Pío García, verdadero soporte de la gestión institucional del Centro, partió a París para realizar estudios de posgrado en Sociología. El segundo es que hacia el último trimestre de 1967, Eduardo Hamuy optó por alejarse del CESO, decisión gatillada en gran medida por los reiterados cuestionamientos que había recibido a propósito de sus encuestas político-electorales (AUCH, 1965 y 1967). De otro lado, su completo desapego de la 'disciplina' universitaria y de las labores administrativas, que se había vuelto del todo notorio tras la partida de García, llevaron al decano Edgardo Boeninger a nombrar provisionalmente a Eduardo Gana Barrientos como director suplente, que pronto fue reemplazado por el sociólogo Laureano Ladrón de Guevara. Estos avatares coincidieron con la 'reforma universitaria' que alteró la vida político-académica de la Universidad de Chile, con especial énfasis a partir de mayo de 1968. Con todo, el hecho de que el personal académico del ceso mantuviese una intensa actividad intelectual, sostén del enorme prestigio alcanzado entre los estudiantes de la Escuela de Economía, explica en gran medida que el Centro se sobrepusiera a tales vaivenes.

DE LA REESTRUCTURACIÓN A LA TRANSICIÓN (1969-1973)

Con Ladrón de Guevara como director se consiguieron 'sortear' las dificultades acrecentadas tras el alejamiento de Hamuy. Y con su decisión, 
hacia mediados de 1969, de realizar estudios de posgrado fuera de Chile se dio paso a la renovación de las autoridades unipersonales del Centro, sobre la base de los mecanismos acordados tras el 'mayo santiaguino' del año anterior, resultó electo como nuevo director del CEso Pío García -ya de regreso-, quien asumió el cargo a partir del $1^{\circ}$ de octubre de 1969.

Con García el Centro experimentó una profunda restructuración que se expresó en la consolidación de una base de investigadores de buen nivel, en la redefinición de las áreas de investigación, el funcionamiento de un seminario general de discusión de proyectos y avances de investigación, la elaboración de una ambiciosa propuesta para la creación de un programa de doctorado -que no llegó a implementarse-, y el perfeccionamiento del programa de publicaciones, a lo que se sumó una sólida gestión institucional que se convirtió en la base de la proyección del Centro más allá de la Universidad.

Entre los nuevos investigadores incorporados al CESO, destaca el sociólogo brasileño Ruy Mauro Marini quien, tras el breve paso de casi un año por la Universidad de Concepción, a fines de 1970 aceptó la invitación para formar parte del cada vez más renombrado grupo de académicos del Centro. Cabe mencionar que a fines de 1969, antes de abandonar su primer exilio en México, Marini había terminado su primer libro, que fue publicado pocos meses después con el título Subdesarrollo y revolución (Marini, 1970).

Resulta igualmente significativa la llegada de Marta Harnecker, quien se desempeñó como coordinadora de seminarios, proveniente de Francia, en donde se había convertido en discípula del filósofo marxista Louis Althusser y que comenzaba a hacerse conocida por la amplia difusión de su libro sobre Los conceptos elementales del materialismo histórico (1968). En su paso por el CESo, Harnecker estuvo a cargo del Seminario de lectura de El Capital de Marx, el que posteriormente fue coordinado por Marini.

Otro de los investigadores que se sumó al CEso fue el economista estadounidense André Gunder Frank. Aunque en octubre de 1968 había sido contratado como profesor en la Escuela de Sociología de la entonces Facultad de Filosofía y Educación de la Universidad de Chile, en el Centro se evaluó detenidamente la posibilidad de su arribo, a condición de que 'atemperara' su explosivo carácter (García, 2012/2013). Esto resultaba particularmente importante para un equipo que -con alguna excepciónhabía conseguido tejer una excelente red de relaciones personales y había 
garantizado espacios para un permanente y fructífero diálogo -al decir de Marini- verdadero secreto de su intensa vida intelectual y del prestigio que alcanzó. El condicionamiento, en modo alguno buscó invalidar el sano debate teórico -e incluso la polémica-con sus compañeros, fue asumido rápidamente por Frank (1971 y 1991). Conocido es que, a esas alturas, Frank era considerado ya un potente intelectual que, con sus ensayos $E l$ desarrollo del subdesarrollo (1966 y 1966b) y Capitalismo y subdesarrollo en América Latina (1967 y 1970), contribuyó a darle un fuerte soporte teórico a la sociología del (sub)desarrollo y a la teoría de la dependencia. Entre las contribuciones de Frank durante su estadía en el CESo, destaca la publicación de su libro Lumpenburguesía: Lumpendesarrollo. Dependencia, clase y política en Latinoamérica (1971).

A los anteriores investigadores hay que añadir las contrataciones, poco tiempo antes, del historiador Jaime Torres y del economista Cristián Sepúlveda, quienes también hicieron destacadas contribuciones a los estudios sobre la dependencia (Torres, 1972; Sepúlveda, 1972 y 1973).

En relación con la reestructuración del Centro debemos señalar que la redefinición de los equipos de investigación se realizó en torno a tres áreas temáticas: la de "Dependencia", dirigida por dos Santos; la de "Estado y Clases Sociales", a cargo de Marini y; la de "Ideología y Cultura", encabezada por Vasconi. Nótese que las tres áreas fueron conducidas por tres de los más destacados dependentistas. Y es que con seguridad la principal contribución teórica del Ceso al pensamiento crítico latinoamericano -y mundial- se relaciona con el análisis de la dependencia.

Especial mención merece en este período la publicación, en octubre de 1970, del libro Chile, hoy-compilado por Víctor Brodersohn-, iniciativa propuesta al CESO por Arnaldo Orfila, director fundador de la editorial Siglo XXI, a fines de 1969, y que tuvo como propósito ofrecer una visión de la realidad chilena que diera cuenta de las transformaciones acaecidas en los últimos decenios y de las perspectivas presentes en sus dominios socioeconómico, político y cultural. Para ese efecto se solicitaron contribuciones a investigadores del Centro así como a destacados académicos chilenos, entre los que cabe mencionar a Jacques Chonchol, Aníbal Pinto, Ariel Dorfman, Enzo Faletto y Eduardo Ruíz (Brodersohn, 1970).

Por otra parte, a fines de abril de 1970 la vida político-académica de la Facultad de Ciencias Económicas se vio fuertemente alterada por la intensa movilización que los estudiantes de izquierda de la Escuela de 
Economía emprendieron -junto con los de Sociología, Psicología e Historia- con el propósito de crear ipso facto una Facultad de Ciencias Sociales. Esa lucha, que a poco de iniciada recibió el apoyo de los investigadores del CEso, y que buscaba poner en cuestión la reforma negociada 'desde arriba', sobrevino en circunstancias en que gran parte de la izquierda universitaria tenía puestas sus energías en las elecciones presidenciales de septiembre de ese año, por lo que pronto se desactivó. Resulta pertinente la mención de este hecho porque una preocupación permanente de los investigadores del CESO se vincula con el debate sobre el rol de las ciencias sociales en las universidades y su relevancia para el proceso de transformación social.

Tras el triunfo de Salvador Allende, candidato de la Unidad Popular (UP), en las elecciones presidenciales del 4 de septiembre de 1970 diversos académicos del ceso pasaron a desempeñar funciones en el nuevo gobierno. Entre ellos, Pío García, Orlando Caputo, Jorge Leiva y Sergio Ramos. Como lo expresa Marini: "En el CEso, eso conllevó la promoción del personal joven... y a la incorporación de nuevos miembros, en la mayoría extranjeros, lo que trajo una gran renovación" (Marini, c1990). De ahí que, en marzo de 1971 y a propuesta de sus colegas, el economista Roberto Pizarro asumió el cargo de director.

En relación con el período abierto tras la victoria de la llamada 'vía chilena al socialismo' nos referimos a cuatro asuntos de la mayor significación para la reconstrucción biográfica del CEso en ese período.

El primero de ellos es la proyección que tuvo el ceso a nivel nacional e internacional, luego de la reestructuración referida. Dicho impulso se expresó, particularmente, en la creación de la revista Sociedad y desarrollo y del semanario Chile HOY. La primera permitió que diversas investigaciones del Centro alcanzaran una amplia difusión, abriéndose un diálogo fructífero con científicos sociales del mundo entero. El prestigio alcanzado, a esas alturas, por Dos Santos, quien estaba a la cabeza de esa iniciativa, permitió que en sus páginas se reprodujeran trabajos de autores como Samir Amir, Michael Löwy, Paul Sweezy, Fernando Henrique Cardoso, Ernesto Laclau, Agustín Cueva y Pablo González Casanova, además de los escritos por sus compañeros en el CESO, por ese entonces también connotados investigadores, a los que últimamente se habían sumado José Valenzuela, Álvaro Briones, José Bengoa y los brasileños Emir Sader y Marco Aurelio García. No obstante, debido a los críticos momentos vividos en la Facultad de Ciencias Económicas, la revista no pasó del tercer 
número; su corta vida es muestra de la intensa producción teórica que tuvo lugar en esos años en el Centro. Por su parte, el semanario Chile HOY -que entre junio de 1972 y septiembre de 1973 publicó sesenta y cinco números-, aunque independiente del CEso, fue una iniciativa organizada por Pío García tras su incorporación al Gobierno. Junto con dos Santos y luego también con Marini, se dieron a la tarea de crear una publicación que acogiera los distintos puntos de vista existentes en la izquierda y en el movimiento popular. Su directora fue Marta Harnecker, el alma de esa iniciativa, quien junto a un pequeño equipo periodístico -en el que destacaron José Cayuela, Gustavo González, Daniel Waksman, Darío Carmona, Víctor Vaccaro y Faride Zerán-, registraban 'en terreno' lo que acontecía en el seno del movimiento popular, al mismo tiempo que consiguieron una llegada privilegiada con las figuras políticas y dirigentes de la época, lo que permitió 'tomarle el pulso' al proceso chileno tanto 'desde arriba' como 'desde el abajo social'.

El segundo se refiere a la disputa abierta en la Facultad de Ciencias Económicas de la Universidad de Chile, en medio de su institucionalizada reforma, tras el frustrado intento de crear una Facultad de Ciencias Sociales. De la fuerte polarización de la política nacional no estuvieron exentas las universidades chilenas y, menos aún, esa politizada Facultad. Si bien ahí el conflicto fue intenso, finalmente se resolvió a través de los mecanismos definidos por la reforma universitaria, oficializándose la división de la Facultad tras la realización de un referéndum. Así, la Facultad de Ciencias Económicas dio paso -a fines de noviembre de 1972- a dos Facultades distintas: una, denominada 'Facultad de Economía Política', que funcionó en el edificio de la hasta entonces Escuela de Economía, y en la que se agrupó la izquierda; la otra, bautizada como 'Facultad de Ciencias Económicas y Administrativas', acogió a los opositores al gobierno de Allende. Con esa decisión, Roberto Pizarro pasó a ser decano de Economía Política y el ceso se convirtió en uno de los departamentos de esa nueva Facultad, en cuya dirección fue nombrado - por consenso de sus académicos- Theotonio dos Santos. Esta situación explica, en gran medida, la interrupción de la revista Sociedad y desarrollo y de la serie Cuadernos del CESO. Sin embargo, esta última reapareció a fines de junio de 1973 con la publicación de dos valiosos ensayos de Bambirra: Capitalismo dependiente latinoamericano y La revolución cubana: una reinterpretación (1973 y 1973b). Por su parte, un mes antes del cruento 11/S, Marini enca- 
bezó un nuevo proyecto editorial, al margen de la Universidad, enfocado a la discusión teórico-política: la revista Marxismo y revolución, que alcanzó a publicar sólo su primer número.

El tercer hecho al que es preciso hacer mención, es el X Congreso de la Asociación Latinoamericana de Sociología (alas) realizado en Santiago entre agosto y septiembre de 1972, en el que hubo una importante presencia del Ceso, destacando las intervenciones de Marini y Frank. Fue la ocasión para que el primero de ellos expusiera su hoy conocido ensayo Dialéctica de la dependencia (Marini 1972b y 1973), que algunos meses antes había sido publicado -aún incompleto- en la revista Sociedad y desarrollo (Marini, 1972) y que fue objeto de una temprana réplica (Cardoso, 1972). La de Frank, como acostumbraba, fue una intervención provocativa y, a la vez, estimulante, más tarde reproducida bajo el impetuoso título: La dependencia ha muerto. Viva la dependencia y la lucha de clases (Frank, 1972 y 1973), y en la que advierte de los límites y dificultades que enfrentaban las 'teorías de la dependencia'.

El cuarto asunto al que deseamos referirnos, es el Simposio sobre Transición al Socialismo y Experiencia Chilena que, en octubre de 1971, organizó el CEso en colaboración con el Centro de Estudios de la Realidad Nacional (CEREN) de la Universidad Católica, y que contó con la participación de connotados científicos sociales, entre los que cabe mencionar a Paul Sweezy, Rossana Rossanda, Michel Gutelman y Lelio Basso, que tuvieron un fructífero intercambio, entre otros, con Franz Hinkelammert, Armand Mattelard, Jacques Chonchol, Pedro Vusković, así como con Frank, Dos Santos, Harnecker y Marini. Se trata de un evento que ilustra el papel protagónico que el CEso desempeñó en el intenso diálogo mundial realizado durante esos años en torno al problema de la transición, así como acerca del importante desafío que la experiencia chilena planteaba a las instituciones académicas, "en el sentido de adaptar su trabajo al proceso social, contribuyendo desde ese ángulo a su comprensión" (CEREN-CEso, 1972). Al respecto, tiempo después, Frank diría:

En el CEso, mi instituto en la Universidad de Chile... Dos Santos, Marini, Pío García, Marta Harnecker y muchos otros debatían los pro y los contra de la transición al socialismo. Yo me hice impopular por mis advertencias de que deberíamos preocuparnos mejor del advenimiento de la reacción y la posible transición al fascismo... Un año más tarde Pinochet bombardeaba el viejo palacio presidencial y dentro de éste al Presidente Constitucional (Frank, 1991: 60-61). 


\section{CLAUSURA, PERSECUCIÓN Y ENJUICIAMIENTO (1973...)}

Con el bombardeo de La Moneda comenzó la persecución de los militantes y simpatizantes de izquierda. En el bando militar núm 10 del mismo día 11 de septiembre, la autodenominada "Junta de Gobierno de las Fuerzas Armadas y Carabineros", conminaba a noventa y cinco dirigentes políticos y sociales a "entregarse voluntariamente" y advertía que la no presentación significaba ponerse al margen de lo dispuesto por la Junta "con las consecuencias fáciles de prever". En aquella lista aparecía un nombre conocido, aunque mal apuntado: "Teotorio Dos Santos", fue escrito en el parte militar.

Ocho días más tarde, el grupo de consejeros opositores a la UP en el Consejo Directivo Superior de la Universidad de Chile, a propuesta del rector Boeninger, acordó la reorganización de varias sedes universitarias y, particularmente, de la Facultad de Economía Política; para ello se decidió suspender temporalmente el semestre académico y designar una Comisión Reorganizadora (AUCH, 1973). Con la decisión de la Junta militar, a inicios del mes de octubre, de intervenir las universidades y nombrar rectores castrenses, se consumó el cierre definitivo de la Facultad de Economía Política y del ceso.

Luego del golpe militar la casona de Av. España 620 y el edificio de Av. República 517 fueron asaltados por la soldadesca y, al poco tiempo, el último se convirtió en el centro de operaciones de la Central Nacional de Investigaciones (CNI), policía política de la dictadura que en 1977 reemplazó a la Dirección Nacional de Inteligencia (DINA).

La mayoría de los investigadores del Ceso se asiló en alguna legación diplomática. Marini recuerda que "cerca de 200 personas hacían un esfuerzo disciplinado y solidario para coexistir en un pequeño departamento, bajo el ruido de las bombas y tiroteos" (Marini, c1990). Dos Santos permaneció seis meses en la Embajada de Panamá. La casa de José Domingo Cañas 1367, en la que vivía con Bambirra, y que transfirieron a la Embajada de Panamá para albergar a otros perseguidos políticos, más tarde fue ocupada por la DINA y convertida en el siniestro Cuartel Ollagüe, otro centro de secuestro, tortura y desaparición.

Con la clausura definitiva del Ceso, la exoneración de su personal de la Universidad de Chile y el exilio de gran parte de sus investigadores se abrió una nueva etapa para la teoría de la dependencia. Y 1974 fue un año crucial, porque en el xi Congreso de la ALAS realizado en Costa Rica 
tuvieron lugar trascendentales debates sobre el alcance y la pertinencia de ese esfuerzo teórico (Camacho, 1979). Fue también durante ese año cuando gran parte de los dependentistas del CEso se reencontró en México, en un segundo o tercer exilio, según los casos. A partir de ese desembarco comenzaron renovados desafíos para ese grupo de teóricos sociales críticos. Junto con la preocupación por seguir dilucidando los mecanismos que reproducen la dependencia y el subdesarrollo, debieron esforzarse por explicar las causas del descalabro del movimiento popular chileno y por cimentar diversos instrumentos para la batalla político-ideológica contra las dictaduras militares de la región; otra historia.

BIBLIOGRAFÍA

AUCH (1965, 1967 y 1973); Actas del Consejo Universitario y del Consejo Directivo Superior de la Universidad de Chile. Archivo Andrés Bello de la Universidad de Chile: sesiones del 27/01/1965, 12/04/1967 y 19/09/1973.

BAMBIRRA, V. (1973); Capitalismo dependiente latinoamericano (Cuadernos del Centro de Estudios Socioeconómicos, 16). Santiago de Chile: CESO-PLA (Prensa Latinoamericana S.A.).

(1973b); La revolución cubana: una reinterpretación (Cuadernos del Centro de Estudios Socioeconómicos, 18). Santiago de Chile: CESO-PLA.

BRODERSOHN, V, comp. (1970); Chile, hoy. México: Siglo XXI Editores.

CAMACHO, C. (1979); Debates sobre la teoría de la dependencia y la sociología latinoamericana. San José de Costa Rica: Editorial EDUCA.

CARDOSO, F. H. (1972); "Notas sobre el estado actual de los estudios sobre dependencia", en Revista Latinoamericana de Ciencias Sociales, 4, 3-31.

CARMAGNANI, M. (1971); Sviluppo industriale e sottosviluppo economico. Il caso cileno (1860-1920). Torino: Fondazione Luigi Einaudi.

(1998); Desarrollo industrial y subdesarrollo económico, El caso chileno (1860-1920). Santiago de Chile: Dirección de Bibliotecas, Archivos y Museos (DIBAM).

CAPUTO, O. \& PIZARRO, R. (1971); Imperialismo, dependencia y relaciones económicas internacionales (Cuadernos del Centro de Estudios Socioeconómicos, 12/13). Santiago de Chile: CESO.

CESO (1967); "Investigación sobre relaciones de dependencia en América Latina", en Boletín del Centro de Estudios Socioeconómicos, 1, 2.

CEREN-CESO (1972); Transición al socialismo y experiencia chilena. Santiago de Chile: CEREN-CESO-PLA. 
COMBLIN, J. (1978); El poder militar en América Latina. La ideología de la Seguridad Nacional. Salamanca: Sal Terrae.

DESCOUVIĖRES, C. (1968); Alcoholismo y familia. Un estudio exploratorio (Cuadernos del Centro de Estudios Socioeconómicos, 9). Santiago de Chile: CESO.

FRANK, A. G. (1966); "El desarrollo del subdesarrollo”, en Desarrollo Indoamericano, 1(2), 13-16.

(1966b); “The development of underdevelopment”, en Monthly Review, 18(4), 17-31.

(1967); Capitalism and Underdevelopment in Latin America. New York: Monthly Review Press.

(1970); Capitalismo y subdesarrollo en América Latina. Buenos Aires: Siglo XXI Editores.

(1971); Lumpenburguesía: Lumpendesarrollo. Dependencia, clase y política en Latinoamérica. Santiago de Chile: CESO-PLA.

(1972); “De la dependencia hacia la acumulación”, en Problemas del Desarrollo, 13, 19-44.

, (1973); "La dependencia ha muerto. Viva la dependencia y la lucha de clases. Una respuesta a críticos”, en Desarrollo Económico, 13(49), 199219.

, (1991); El subdesarrollo del desarrollo. Un ensayo autobiográfico. Caracas: Editorial Nueva Sociedad.

GARCÍA, P. (1980); “Concepciones económicas y acción política; consideraciones sobre la experiencia chilena”, en Problemas del desarrollo, 42, 79-89.

(2012/2013); Entrevistas con el autor. Ciudad de México / Belgrado, Serbia.

GÓNGORA, M. (1966); Vagabundaje y sociedad fronteriza en Chile (Siglos XVII a XIX) (Cuadernos del Centro de Estudios Socioeconómicos, 2). Santiago de Chile: CESO.

HAMUY, E. (1965); “Historiar el presente”, en Memoria del Seminario realizado en el Instituto de Economía de la Universidad de Chile, 80, 105-110.

, (1966); Temas de nuestro tiempo (Cuadernos del Centro de Estudios Socioeconómicos, 1). Santiago de Chile: CESO.

(1967); Chile: el proceso de democratización fundamental (Cuadernos del Centro de Estudios Socioeconómicos, 4). Santiago de Chile: CESO.

HERNÁNDEZ, S. (1966); Transformaciones tecnológicas en la agricultura de Chile Central. Siglo XIX (Cuadernos del Centro de Estudios Socioeconómicos, 3). Santiago de Chile: CESO.

HARNECKER, M. (1968); Los conceptos elementales del materialismo histórico. México: Siglo XXI Editores. 
IZQUIERDO, G. (1968); Un estudio de las ideologías chilenas (La Sociedad de Agricultura en el siglo XIX). Santiago de Chile: Imprenta Técnica Ltda.

KAY, C. (1980): El sistema señorial europeo y la hacienda latinoamericana. México: Ediciones ERA, Serie popular.

LADRÓN DE GUEVARA, L. (1967); Propietario y Empresario Agrícola (Algunas de sus características en el caso de Aconcagua) (Cuadernos del Centro Socioeconómico, 5). Santiago de Chile: CESO.

MARINI, R. M. (1970); Subdesarrollo y revolución. México: Siglo XXI Editores.

(1972); "Dialéctica de la dependencia: una economía exportadora", en Sociedad y desarrollo, 1, 35-51.

, (1972b); Dialéctica de la dependencia (Documento de Trabajo). Santiago de Chile: CESO.

, (1973); Dialéctica de la dependencia. México: Ediciones ERA.

(c1990); Memoria. Recuperado el 15 de junio de 2013, de http:// www.marini-escritos.unam.mx

RAMOS, S. (1972); Chile: ¿una economía de transición? (Cuadernos del Centro Socioeconómico, 15). Santiago de Chile: CESO-PLA.

SÁNCHEZ, A. (1976); Cultura y revolución: un ensayo sobre Lenin. México: Ediciones ERA, Serie Popular.

SANTOS, T. (1967); El nuevo carácter de la dependencia (I). Gran Empresa y Capital Extranjero (Cuadernos del Centro de Estudios Socioeconómicos, 6). Santiago de Chile: CESO.

, \& BAMBIRRA, V.; CAPUTO, O.; MARTÍNEZ, J.; PIZARRO, R. \& RAMOS, S. (1968); Imperialismo y dependencia externa: resumen y discusión de las principales teorías (Documento de Trabajo). Santiago de Chile: CESO.

(1968b); El nuevo carácter de la dependencia (Cuadernos del Centro de Estudios Socioeconómicos, 10). Santiago de Chile: CESO.

, (1968c); "Crisis de la Teoría del Desarrollo y las relaciones de dependencia en América Latina”, en Boletín del Centro de Estudios Socioeconómicos, 3, 2-40.

\& BAMBIRRA, V.; CAPUTO, O.; MARTÍNEZ, J.; PIZARRO, R. \& RAMOS, S. (1969); Bibliografía para la investigación sobre relaciones de dependencia en América Latina (Documento de Trabajo). Santiago de Chile: CESO.

, (1969b); Socialismo o fascismo: dilema latinoamericano. Santiago de Chile: CESO-PLA.

, (1970); Dependencia y cambio social (Cuadernos del Centro de Estudios Socioeconómicos, 11). Santiago de Chile: CESO. 
SCHEJTMAN, A. (1970); Peasant Economies within the Large Haciendas of Central Chile (thesis). Oxford, England: University of Oxford.

SEPÚlVEDA, C. (1972); Dos modelos de acumulación de capital en el desarrollo capitalista chileno (Memoria de Prueba), Universidad de Chile, Santiago de Chile.

, (1973); Desarrollo económico en Chile (Documento de Trabajo). Santiago de Chile: CESO.

TORRES, J. (1972); Para un concepto de "formación social colonial" (Documento de Trabajo). Santiago de Chile: CESO.

VALENZUELA, J. (1974); El capitalismo en el centro: problemas actuales. Caracas: Universidad Central de Venezuela, Facultad de Ciencias Económicas y Sociales.

VASCONI, T. A. (1968); Educación y cambio social (Cuadernos del Centro de Estudios Socioeconómicos, 8). Santiago de Chile: CESO.

(1970); Dependencia y superestructura (Documento de Trabajo). Santiago de Chile: CESO.

, \& RECA, I. C. (1971); Modernización y crisis en la universidad latinoamericana (Cuadernos del Centro Socioeconómicos, 14). Santiago de Chile: CESO.

ANEXO:

PublicACIONES dEL CENTRO DE Estudios SOCIOECONÓMICOS (1966-1973)*

A. Publicaciones colectivas del Ceso:

PUBLICACIONES PERIÓDICAS:

Boletín del Centro de Estudios Socioeconómicos (1967-1968): núm. 1 al 3.

Cuadernos del Centro de Estudios Socioeconómicos (1966-1973): núm. 1 al 18 (excepto el $\mathrm{n}^{\circ} 17$, que no alcanzó a ser publicado).

Revista Sociedad y desarrollo (1972): $\mathrm{n}^{\circ} 1$ al 3.

LIBROS:

Chile, hoy (1970). México: Siglo XXI Editores.

Transición al socialismo y experiencia chilena (1972). Santiago de Chile: CESO-CEREN / PLA.

* No incluimos las obras de los investigadores del ceso ya referidas en la bibliografía. 


\section{B. OTRAS PUBLICACIONES CREADAS POR INVESTIGADORES DEL CESO:}

\section{PUBLICACIONES PERIÓDICAS:}

Semanario Chile HOY (1972-1973): núm. 1 al 65.

Revista Marxismo y revolución (1973): núm. 1.

\section{Publicaciones de inVestigadores DEL CESO:}

\section{COEDICIÓN CESO-PLA:}

BAMBIRRA, V. (1971); Diez años de insurrección en América Latina (tomos I y II). Santiago de Chile: CESO- PLA, Colección América Nueva.

FRANK, A. G. (1968); Chile: el desarrollo del subdesarrollo. Santiago de Chile: CESO-PLA, Colección América Nueva.

SANTOS, T. (1971); La crisis norteamericana y América Latina. Santiago de Chile, CESO-PLA, Colección América Nueva.

(1972); Socialismo o fascismo: el nuevo carácter de la dependencia y el dilema latinoamericano. Santiago de Chile: CESO-PLA, Colección América Nueva.

(1973); Imperialismo y corporaciones multinacionales. Santiago de Chile: CESO-PLA, Colección América Nueva.

\section{DOCUMENTOS DE TRABAJO (MIMEOGRAFIADOS):}

BAMBIRRA, V. (1971); Hacia una tipología de la dependencia: industrialización y estructura socio-económica (Documento de Trabajo). Santiago de Chile: CESO.

(1972); Las estructuras dependientes en la fase de la integración monopólica mundial. Contradicciones del capitalismo dependiente (Documento de Trabajo). Santiago de Chile: CESO.

BRIONES, A. (1972); Los conglomerados transnacionales y la integración del sistema capitalista mundial: el caso chileno (Documento de Trabajo). Santiago de Chile: CESO.

MARINI, R. M. (1971); El subimperialismo brasileño (Documento de Trabajo). Santiago de Chile: CESO.

, (1972); La acumulación capitalista dependiente y la superexplotación del trabajo (Documento de Trabajo). Santiago de Chile: CESO. 
QUIJANO, A. (1970); Redefinición de la dependencia y marginalización en América Latina (Documento de Trabajo). Santiago de Chile: CESO.

RAMOS, S. (1972); La independencia del desarrollismo (Documento de Trabajo). Santiago de Chile: CESO.

VALENZUELA, J. (1972); Desarrollo capitalista, socialización y revolución: el modelo clásico (Documento de Trabajo). Santiago de Chile: CESO.

, (1972); El problema del trabajo productivo e improductivo en los clásicos y en Marx (Documento de Trabajo). Santiago de Chile: CESO.

\section{EN OTRAS EDITORIALES Y REVISTAS:}

CAPUTO, O. \& PIZARRO, R. (1970); Desarrollismo y capital extranjero. Las nuevas formas del imperialismo en Chile. Santiago de Chile: Ediciones de la Universidad Técnica del Estado (UTE).

CAPUTO, O.; FRANK, A. G.; PIZARRO, R. \& QUIJANO, A. (1973); Aspectos de la realidad latinoamericana. Santiago de Chile: Empresa Editora Nacional Quimantú, Colección Camino Abierto.

FRANK, A. G. (1970); “Dependencia económica, estructura de clases y política del subdesarrollo en Latinoamérica”, en Revista Mexicana de Sociología, 32(2), 229-282.

RAMOS, S. (1972); Chile: ¿una economía de transición? La Habana: Casa de las Américas, Colección Premio Casa de las Américas.

SANTOS, T. (1970); Lucha de clases y dependencia en América Latina. Medellín: Editorial La Oveja Negra.

(1973); Dependencia económica y cambio revolucionario en América Latina. Caracas: Editorial La Enseñanza Viva, Colección de Bolsillo.

VASCONI, T. A. \& LESSA, C. (1969): Hacia una crítica de las interpretaciones del desarrollo latinoamericano. Caracas: Centro de Estudios del Desarrollo / Universidad Central de Venezuela.

VASCONI, T. A. (1968); “Cultura, dependencia, ideología y alienación”, en Revista Mexicana de Sociología, 30(4), 819-837.

(1970); Dependencia y superestructura y otros ensayos (sobre ideología y educación en América Latina). Caracas: Ediciones de la Biblioteca de la Universidad Central de Venezuela, Colección Avance.

, (1972); Ideología, lucha de clases y aparatos educativos en el desarrollo de América Latina. Bogotá: Editorial Latina, Colección Temas Latinoamericanos. 